\title{
Herkunfts-, Ethno- und Weltorientierte: Aneignungstypen der kulturellen Identität und kommunikativen Vernetzung in der Diaspora
}

\author{
Andreas Hepp / Cigdem Bozdag / Laura Suna
}

Auf der Basis einer medienethnografischen Untersuchung u. a. mittels qualitativer Interviews und Netzwerkanalysen werden in diesem Artikel für die marokkanische, russische und türkische Diaspora in Deutschland drei Typen des Wechselverhältnisses von kultureller Identität und kommunikativer Vernetzung in der Medienaneignung unterschieden, nämlich „Herkunftsorienierte“, „Ethnoorientierte“ und „Weltorientierte“. Die Form der kommunikativen Vernetzung artikuliert sich zusammen mit der jeweils subjektiv empfundenen kulturellen Zugehörigkeit als „Herkunftsvernetzung “, „, bikulturelle Vernetzung " und „transkulturelle Vernetzung“. Solche Analysen machen deutlich, dass eine Reduktion der Forschungsdiskussion auf die "Integrationsproblematik" nicht zielfübrend ist. Im Gegenteil sind die Potenziale, die in der kommunikativen Vernetzung von Migrantinnen und Migranten mittels unterschiedlicher Medien bestehen, weit vielfältiger.

Schlagwörter: Migration, Diaspora, Medien, Identität, Integration, Vernetzung, Medienkultur, Netzwerkanalyse, Grounded Theory

\section{Einleitung: Medien und Migration überdenken}

Die Kommunikations- und Medienwissenschaft diskutiert im deutschen Sprachraum Fragen der Medien(-nutzung) und Migration primär im Rahmen einer nationalkulturellen Integration. ${ }^{1}$ Während hierbei wichtige Forschungsergebnisse erarbeitet wurden, ist dieser Zugang doch verkürzend, wenn er die national-integrative Rahmensetzung als einzigen Bezugspunkt begreift. Mit fortschreitender Globalisierung, die sich u. a. in (Trans-)Migration und grenzüberschreitender (digitalisierter) Kommunikation sowie damit zusammenhängend - in weitergehender kultureller Differenzierung von Großregionen und Staaten konkretisiert, ist die Lage wesentlich vielschichtiger geworden. Versteht man unter Migration die alltagsweltlich relevante, großräumige Verlagerung des Lebensmittelpunkts durch lokale Mobilität (Oswald 2007: 17f.), können wir Migration nicht (mehr) als einen linearen Wanderungsprozess von einem Herkunfts- in ein Aufnahmeland begreifen, in das sich Menschen akkulturalisieren bzw. assimilieren, wofür Medien ggf. eine Stütze wären - falls ein solcher Blickwinkel jemals zielführend gewesen ist. Vielmehr ist Migration selbst hochgradig differenziert. Migrantinnen und Migranten leben verschiedene Formen von Identitäten und kulturellen Zugehörigkeiten, für deren Artikulation „traditionelle“ Massenmedien (Fernsehen, Film, Zeitung, Radio etc.) und „neue“ digitale Medien (WWW, Social Web, (Mobil- und Internet-)Telefon etc.) eine wichtige Ressource bilden. In der internationalen Forschungsdiskussion hat sich diesbezüglich das Konzept der „Diaspora“ etabliert (Dayan 1999; Georgiou 2006). Mit die-

1 Exemplarisch sei auf eine Reihe von jüngeren Überblickswerken verwiesen wie bspw. Schatz et al. 2000; Geißler/Pöttker 2006 oder Bonfadelli/Moser 2007. Zur Problematisierung des Integrationskonzepts in der Migrationsforschung im Allgemeinen siehe Favell 2003 und die Beiträge in Hess et al. 2009. 
sem Konzept wird verbunden, dass Migrantinnen und Migranten als Gruppe spezifische Sozialfigurationen bzw. Vergemeinschaftungen konstituieren, die nicht einfach dem Leben der Identität einer „Herkunftsnation“ in der Fremde entsprechen. Aus kommunikations- und medienwissenschaftlicher Perspektive lässt sich formulieren, dass diese intern vielfältig differenzierten Diasporas in dem Sinne „deterritorial“ (Hepp 2006: 282) sind, dass sie sich - bei einem geteilten Sinnhorizont der Migrationsvergemeinschaftung - als Vernetzung verschiedener lokaler Gruppen über unterschiedliche Territorien hinweg erstrecken. Für die Aufrechterhaltung dieser Sozialfiguration sind Medien wegen ihres deterritorialen Kommunikationspotenzials eine wichtige Stütze.

Solche Vorüberlegungen aufgreifend wollen wir uns in diesem Aufsatz auf der Basis der Forschungsergebnisse eines von der Deutschen Forschungsgemeinschaft geförderten Projekts ${ }^{2}$ mit dem Stellenwert von Medien für Migrantinnen und Migranten auseinandersetzen. Im Kern befassen wir uns mit der Fragestellung, wie sich kulturelle Identitäten von Migrantinnen und Migranten mit deren kommunikativer Vernetzung im Prozess der Medienaneignung artikulieren - letzterer verstanden als ein aktiver Vorgang des Sich-zu-Eigen-Machens von Medien und ihren Inhalten. Unser Ziel ist es konkreter, die Beziehung von kultureller Identität und kommunikativer Vernetzung für die marokkanische, russische und türkische Diaspora in Deutschland typologisierend zu erfassen. Ein solches Vorgehen versucht zuerst einmal, offener bei der alltagsweltlichen Perspektive auf die Beziehung von Migration und Medienaneignung anzusetzen: Es geht uns darum, das Wechselverhältnis zwischen bestimmten Identitätsorientierungen und einer kommunikativen Vernetzung mittels unterschiedlicher Medien in ihrer Gesamtheit zu erfassen, ohne zwei implizite Setzungen der „Medien- und Integrationsforschung“ zu replizieren. Dies ist einerseits die Setzung, Migrationsgemeinschaften bzw. Diasporas seien als eine in sich homogene Gruppe „Anderer“ beschreibbar, andererseits die Setzung, bei einer Betrachtung der Mediennutzung von Migrantinnen und Migranten gehe es primär oder ausschließlich um die Frage, welchen Beitrag diese Medien „für eine Integration in die deutsche Gesellschaft/Kultur“ leisten. Aus alltagsweltlicher Perspektive sind Fragen der kulturellen Identität und der kommunikativen Vernetzung in ihrer Wechselbeziehung wesentlich vielfältiger. Entsprechend verstehen wir die vorgeschlagene Typologie auch nicht als Beschreibung von essenziell gedachten ethnischen Entitäten, sondern als musterhafte Betrachtung des Gesamtzusammenhangs von kultureller Identität und kommunikativer Vernetzung. Wie dieser Gesamtzusammenhang normativ im Hinblick auf „Integration“ zu werten ist, erscheint uns eine separat zu diskutierende Frage.

Diese Überlegungen vorausgesetzt, argumentieren wir in folgenden Einzelschritten: Zuerst gehen wir auf bestehende Typologien in dem Forschungsfeld Medien und Migration ein. Diese sind aus unserer Perspektive dadurch verkürzend, dass sie a priori den Rahmen einer „nationalen Integration“ setzen. Dem stellen wir unser offenes empirisches Vorgehen entgegen, über das wir zu einer Dreiertypologie von herkunfts-, ethnound weltorientierten Migranten gelangt sind. Nach einer Darstellung dieser materialbasierten Typenbildung betrachten wir deren Konkretisierungen über verschiedene Migrationsgemeinschaften hinweg. Auf der Basis solcher Analysen werden wir abschließend die Relevanz unserer Forschung für eine wissenschaftliche Auseinandersetzung mit normativen Fragen von „Medien und Integration“ diskutieren.

2 Das Forschungsprojekt wird von den Autoren dieses Beitrags unter dem Titel „Integrationsund Segregationspotenziale digitaler Medien am Beispiel der kommunikativen Vernetzung von ethnischen Migrationsgemeinschaften“ (2008-2010) am IMKI, Universität Bremen, realisiert. 


\section{Von der Minderheit zur Diaspora: Nutzertypologien in der Forschung zu Medien und Migration}

Im Forschungsfeld zu Medien und Migration sind verschiedene Typologien von Nutzerinnen und Nutzern etabliert (siehe überblickend bspw. Piga 2007). Die Zahl dieser Typologien ist so groß, dass sie den Rahmen dieser Darstellung sprengen würde. Vor diesem Hintergrund haben wir uns auf solche Typologien konzentriert, die die Diskussion um die uns interessierenden Migrationsgruppen prägen, nämlich die der marokkanischen, türkischen und russischen Diaspora im deutschsprachigen Raum. Unter diesen sind es insbesondere türkische Migrantinnen und Migranten, auf deren Mediennutzung bezogen verschiedene Typologien entwickelt wurden. Im Fokus unserer Betrachtung stehen dabei die normativen Implikationen der Differenzierungskriterien dieser Typologien.

Bisherige Typologien können im positiven Sinne eine Varianz unterschiedlicher Nutzungstypen zeigen, die einem stereotypen Bild des „Medienghettos“, d. h. der kommunikativen Abschottung von Migrantinnen und Migranten zuwiderläuft (siehe auch Simon 2007). Kritisch ist unseres Erachtens an diesen Studien aber zu sehen, dass trotz der Vielfalt der mitunter beschriebenen Typen diese Differenziertheit von vornherein mit Bezug auf eine Aufnahmegesellschaft als Varianz von Integrationstypen gedeutet wird. Im weitesten Sinne wird Integration dabei nicht gesehen als Assimilation, sondern als „der erwünschte soziale Prozess, der die Teile einer Gesellschaft (Individuen, Institutionen, Gruppen) unter Mitwirkung ihres Bewusstseins mehr oder weniger stark zum Ganzen dieser Gesellschaft verbindet“ (Pöttker 2005: 40f.), ein Prozess, der auch über (Medien-)Kommunikation vermittelt ist.

Um dieses Diskursschema in „Reinform“ greifbar zu machen, möchten wir zuerst eine Typologie aufgreifen, die sich nicht auf den deutschen Sprachraum bezieht, die in der Form ihrer Argumentation aber auch für diesen als exemplarisch begriffen werden kann. ${ }^{3}$ Dies ist die Studie von Hanna Adoni, Akiba Cohen und Dan Caspi (Adoni et al. 2006, 2002) zur Nutzung von Fernsehen, Radio und Zeitung durch arabische und russische Personen in Israel. Auf Basis einer standardisierten Telefonumfrage unterscheiden die Autoren vier Typen von Mediennutzern, die über eine Matrix entlang zweier Dichotomien gewonnen werden (Adoni et al. 2002: 423f.): einerseits die Dichotomie von hohem und niedrigem Konsum von hebräischsprachigen Medien, andererseits die Dichotomie von hohem und niedrigem Konsum von herkunftssprachlichen Medien. Sprache wird damit zum primären Differenzkriterium folgender Typen (Adoni et al. 2002: 419f.):

- Dualisten („dualists“) als umfassende Nutzer sowohl von Medien der „Minderheitensprache“ als auch der „Mehrheitensprache“;

- Anpasser („adapters“) als umfassende Nutzer der Medien der „Mehrheitensprache" und geringe Nutzer von Medien der "Minderheitensprache“;

- Separatisten („separatists“) als umfassende Nutzer der Medien der „Minderheitensprache" und geringe Nutzer von Medien der „Mehrheitensprache“;

- Abgekoppelte („detached“) als geringe Nutzer von Medien beider Sprachen.

Während diese Typologie erst einmal normativ offen zu sein scheint, indem sie sich mit der Beziehung von Sprache der genutzten Medien und kultureller Identität befasst, steht in ihrem Zentrum doch die Betrachtung des Beitrags von Medien für eine (soziale und

3 Priska Bucher und Heinz Bonfadelli (2007) bspw. greifen direkt die Forschung von Adoni et al. auf und unterscheiden bei der Mediennutzung von migrantischen Jugendlichen in der Schweiz „Ungebundene“, „Schweiz-Orientierte“, „Herkunfts-Orientierte“ und „Dualisten“. 
politische) Integration in die Aufnahmegesellschaft, wie allein schon die Benennung dieser Typen zeigt. Integration wird dabei als positive Relationierung zur Identität der Mehrheitsbevölkerung des Nationalstaats begriffen. Hierfür wird die Nutzung von Medien sowobl der Herkunftssprache als auch der der Migrationsgesellschaft als zielführend beschrieben, indem eine solche doppelte Nutzung es ermöglicht, die eigene kulturelle Identität aufrechtzuhalten und diese gleichzeitig in Bezug zur Identität der Mehrheit der Aufnahmegesellschaft zu setzen: ${ }^{4}$, the choice of the language in which one uses the media constitutes a social act related to the construction of social identities of minority members" (Adoni et al. 2002: 432).

Es ist eine solche rahmende Argumentationsfigur, die in unterschiedlichen Graden auch die bestehende Typenbildung in Bezug auf Deutschland prägt. Exemplarisch wollen wir dies an den Typologien von Hans-Jürgen Weiß und Joachim Trebbe, Kai Hafez sowie der Darstellung migrantischer Sinus-Milieutypen von Walter Klingler und Albrecht Kutteroff deutlich machen. In der Varianz des ihnen zugrundeliegenden methodischen Vorgehens (sowohl standardisiert als auch nicht-standardisiert) bzw. ihrer Gesamtanlage (kausalistisch-prüfend vs. verstehend-erklärend) können diese Typologien insgesamt als kennzeichnend für den aktuellen Diskussionsstand begriffen werden.

Unter den standardisierten Untersuchungen der letzten Jahre ist zuerst einmal die Typologie von Hans-Jürgen Weiß und Joachim Trebbe (2001) zu erwähnen, die diese als Teil einer Studie zur Mediennutzung und Integration der türkischen Bevölkerung in Deutschland im Auftrag des Presse- und Informationsamtes der Bundesregierung entwickelten. Ausgehend von einer repräsentativen Face-to-Face-Befragung der türkischen Mehrheitsbevölkerung ab 14 Jahren führten Weiß und Trebbe eine hierarchische Clusteranalyse durch. Das Ergebnis der Untersuchung sind sechs „Integrationstypen“ (Weiß/Trebbe 2001: 43): erstens junge Ledige mit hohem Integrationsstatus, zweitens gut integrierte gebildete Berufstätige, drittens junge, berufstätige, gut integrierte Singles, viertens religiöse Verheiratete mit Integrationspotenzial, fünftens schlecht integrierte Frauen ohne Schulbildung und schließlich sechstens religiöse ältere Männer ohne Integrationspotenzial. Ausgehend von dieser Integrationstypologie wird dann die Mediennutzung erfasst, wobei folgende „Grundtendenz" ausgemacht wird: „Je höher der Integrationsgrad, desto größer die Affinität zu deutschsprachigen Medien“ (Weiß/Trebbe 2001: 47) Mit unterschiedlichen Daten und Ausgangspunkten findet sich entsprechend der von Adoni et al. bekannte Ansatz der Annäherung an eine Typologisierung im Hinblick auf Integrationsfragen.

Ein solcher Diskurs durchzieht ebenfalls die Typenbildung in anderen Teilen der standardisierten Mediennutzungsforschung, wie das Beispiel der Sinus-Studie „Lebenswelten von Migranten in Deutschland“ verdeutlicht, als deren Teil die Auswertung „Migrantenmilieus und Medienverhalten“ (Klingler/Kutteroff 2009) realisiert wurde. Dort werden acht Milieus in vier Milieubereichen unterschieden, nämlich die „ambitionierten Migrantenmilieus“ („multikulturelles Performermilieu“ und „intellektuell-kosmopolitisches Milieu“), die „bürgerlichen Migrantenmilieus“ („adaptiv-bürgerliches Milieu“ und „statusorientiertes Milieu“), die „prekären Migrantenmilieus“ („hedonistisch-subkulturelles Milieu“ und „entwurzeltes Milieu“) sowie die „traditions-verwurzelten Migrantenmilieus“ („traditionelles Arbeitermilieu“ und „religiös-verwurzeltes Milieu“) (Klingler/Kutteroff 2009: 299f.). Während diese Untersuchung bemerkenswerte Ergeb-

4 Etwas unklar bleibt allerdings, ob „Abkopplung“ („detached“) wirklich als Ausschluss von Öffentlichkeit gewertet werden kann. Wie Couldry et al. (2007) zeigen, ist die Nutzung bestimmter Medien immer nur eine Möglichkeit der „public connection“, die nicht verabsolutiert werden sollte. 
nisse zur Mediennutzung in einzelnen Migrantenmilieus liefert und $\mathrm{u}$. a. zeigen kann, dass das Herkunftsland nicht die Milieuzugehörigkeit determiniert bzw. dass in den ambitionierten Migrantenmilieus eine kulturelle Orientierung weit über die Grenzen von Herkunfts- und Migrationsland hinaus ausgemacht werden kann, bleibt die Gesamtbewertung der Ergebnisse dem bisher skizzierten Interpretationsraster verhaftet. Es geht letztlich um Fragen der Integration der einzelnen Milieus in eine deutsche Mehrheitsgesellschaft. Zugespitzt formuliert kann man zum „Medienverhalten“ über die Milieus hinweg sagen, dass Fernsehen bzw. eine bi-linguale Mediennutzung allgemein üblich sind. Im Hinblick auf digitale Medien fällt auf, dass diese in den ambitionierten und bürgerlichen Migrantenmilieus überdurchschnittlich, in den anderen Milieus unterdurchschnittlich verbreitet sind. Diese und weitere Einzelergebnisse einbeziehend kommt die Untersuchung zu dem Schluss, dass „[v]or allem in den Milieus der Unterschicht [...] Integrationsvorbehalte und -barrieren“ existieren, die sich auch in der Mediennutzung konkretisieren. Die „modernen Milieus sind dagegen (mit durchaus bikulturellem Selbstbewusstsein) in einer gemeinsamen Gesellschaft angekommen“ (Klingler/Kutteroff 2009: 307).

Differenzierter wird in qualitativen Typologien argumentiert. Exemplarisch dafür kann die ebenfalls im Auftrag des Presse- und Informationsamtes der Bundesregierung realisierte Studie von Kai Hafez angesehen werden. Kai Hafez (2002: 15) greift in seiner qualitativen, auf 93 Tiefeninterviews basierenden Studie zur türkischen Mediennutzung in Deutschland die Forschungstradition der Cultural Studies auf und kritisiert ausgehend davon die beschriebene, von Joachim Trebbe und Hans-Jürgen Weiß (2001) entlang von allgemeinen Integrationskriterien (Sprachkenntnisse, Vertrauen in die deutsche Politik usw.) entwickelte Typologie von Integrations- und Mediennutzertypen. Hafez operiert mit der Sprache als zentralem Ausgangskriterium der Typenbildung, ergänzt um das weitere Kriterium der Verhaftung in „zwei subjektiv empfundenen politischen und kulturellen Sphären des Diskurses - nämlich der türkischen und der deutschen Sphäre“ (Hafez 2004: 15). Im Ergebnis unterscheidet Hafez drei Typen der Nutzung nur türkischer Medien, den „Kulturexil-Nutzer“ (Nutzung, um den Kulturkontakt zu wahren), den „politischen Exil-Nutzer“ (Nutzung, um den national-politischen Kontakt zu wahren) und den „Diaspora-Nutzer“ (Nutzung aus pragmatischen Gründen, bspw. der mangelnden deutschen Sprachkompetenz). Die Aneignung deutscher und türkischsprachiger Medien ist für den „Bikultur-Nutzer“ kennzeichnend, von nur deutsch-türkischen Medien für den „Transkultur-Nutzer“ und von nur deutschen Medien für den „Assimilationsnutzer“. Während diese Typologie einmal mehr ein Verständnis der Vielfalt von Medienaneignung in der türkischen Migrationsgemeinschaft bietet, bleibt auch diese gleichwohl durch Fragen der nationalen Integration gerahmt. So scheint nach $\mathrm{Ha}-$ fez die Mediennutzung „ein Indikator der kulturellen und sprachlichen Integration zu sein - nicht aber der sozialen und politischen“ (Hafez 2004: 34). Aus den Gesamtbefunden folgert er, dass „Integration weitaus eher die Voraussetzung für als die Folge von deutscher Mediennutzung ist“ (Hafez 2004: 35).

Solche exemplarisch ausgewählten Studien und ihre Typologien ${ }^{5}$ bieten einen wichtigen empirischen Einblick in die Nutzung und Aneignung von (Massen-)Medien durch Migrantinnen und Migranten, gerade da, wo das Differenzierungskriterium der Sprache zusätzlich um das der kulturellen Orientierung resp. Identität erweitert wird. Kritikwürdig bleibt gleichwohl, dass mit der tendenziell starren Gegenüberstellung von Medien der Herkunfts- bzw. Aufnahmesprache einerseits und der Rahmung der Forschung

5 Ähnliche Typologien finden sich beispielsweise bei Trebbe 2007. 
durch Fragestellungen der nationalen Integration in die Migrationsgesellschaft andererseits ein linearer Bewertungsblickwinkel in solchen Typologien dominiert. Selbst dort, wo - wie beispielsweise bei der Sinus-Milieu-Differenzierung - die Daten andere Interpretationsmöglichkeiten gestatten würden, geschieht die Deutung der verschiedenen Typen primär im Hinblick darauf, inwieweit diese auf eine „problematische“ oder eine „unproblematische“ Integration in das Migrationsland verweisen, das als Aufnahmegesellschaft konzeptionalisiert wird.

Einer solchen Herangehensweise steht die jüngere Forschung zu Migration und Medien gegenüber. So hat einerseits die soziologische Forschung durch vielfältige Studien gezeigt, dass Migration nicht eine lineare Mobilität in ein definiertes Aufnahmeland bedeutet, sondern mit der „Transmigration“ - also dem nur vorübergehenden Aufenthalt in einem Land, worauf eine weitere lokale Mobilität folgt - auch andere Muster verbreitet sind (siehe bspw. Mau 2007: 43-48; Pries 1998, 2008: 47-59). Andererseits hat vor allem die medienethnografische kommunikations- und medienwissenschaftliche Forschung deutlich gemacht, dass die bestehenden migrantischen Kommunikationsräume vielfältiger sind, als es die bisher diskutierten Typologien nahelegen. Myria Georgiou beispielsweise analysiert in ihrer ethnografischen Untersuchung zur griechisch-zypriotischen Diaspora den besonderen Status von (Groß-)Städten in diasporischen Kommunikationsnetzwerken. Da es insbesondere Großstädte sind, in denen Migrantinnen und Migranten leben, sind ihre Kommunikationsnetzwerke eher als „intercity links“ (Georgiou 2006: 133) zu verstehen und können dennoch nicht von vornherein auf nationale Kommunikationsräume reduziert werden (siehe auch Georgiou 2007: 20; Gumpert/ Drucker 2007). Auf den „diasporic mediated space“ (de Leeuw/Rydin 2007) sind ebenfalls die medienethnografischen Analysen von Sonja de Leeuw und Ingegerd Rydin bezogen. Das Ergebnis ihrer Forschung lässt sich dahingehend zusammenfassen, dass der Kommunikationsraum der Diaspora im Spannungsverhältnis zwischen „Haften“ („bonding“) an den Herkunftskontexten und „Überbrücken“ („bridging“) zu den aktuellen Lebenskontexten steht. Ihres Erachtens sind „alle Medien“ (de Leeuw/Rydin 2007: 192) - angefangen vom Fernsehen bis hin zum Internet - in die Analyse einzubeziehen, wenn man die Differenziertheit dieses Kommunikationsraums angemessen analysieren möchte.

Betrachtet man die letztgenannten Studien in ihrer Gesamtheit, unterstreichen deren Ergebnisse die Notwendigkeit, Fragen von Medien und Migration nicht allein im Rahmen einer nationalen Integration zu sehen. Ganz in diesem Sinne spricht Kevin Robins (2006) von der Sinnhaftigkeit einer transkulturellen Rahmensetzung der Forschung. Er argumentiert, dass die gegenwärtige Migration nach und in Europa dadurch gekennzeichnet ist, dass die Migrantinnen und Migranten nicht mehr - wie in Zeiten postkolonialer Migration - in ein bestimmtes Zielland migrierten, in dem sie dann bleiben. Vielmehr bewegen sie sich zumindest in Teilen über verschiedene Länder hinweg und sind zwischen diesen als Diaspora verstreut. Hieraus folgert er das Potenzial einer spezifischen transkulturellen Konnektivität von Migranten:

„What is distinctive, then, is the nature and degree of transnational connectivity and connectedness between what are variously referred to as „transnational communities“, „transmigrants“, or „new global diasporas“. Migrant populations are connected to each other, and commonly also in close connection to their country of origin. [...] Absolutely crucial here, of course, is the technological and communications infrastructure that now makes this kind of inter-connection possible, and even routine, whether it be cheap and easy air travel or new communications media (for example, satellite television, the Internet). “ (Robins 2006: 25) 
Diese Perspektive der „transkulturellen Vielfalt“ (Robins 2006: 29) in migrantischer Konnektivität versucht, sich analytisch von der Ausschließlichkeit eines Blickwinkels auf Migranten als „national zu integrierende Personen“ zu lösen und in einer größeren Offenheit dem über territoriale Grenzen hinweg ggf. verbindenden Momenten von Diasporas gerecht zu werden. Letztlich geht es uns in unserer Untersuchung darum, einen solchen Zugang mit dem einer typologisierenden Betrachtung von Medienaneignung zu verbinden. Während unseres Erachtens das Potenzial einer typologisierenden Analyse darin besteht, auf systematische Weise die Varianz des migrantischen Umgangs mit Medien über verschiedene Migrationsgruppen hinweg zu erfassen und damit im Sinne Max Webers (1972) erklärend zu verstehen, halten wir es vor dem Hintergrund der aktuellen internationalen Forschung für verkürzend, solche Typologien von vornberein als Integrationstypologien anzulegen. Diese Verkürzung lässt sich durch ein offeneres methodisches Vorgehen vermeiden.

\section{Methodisches Vorgehen: Kulturelle Identität und kommunikative Vernetzung}

Der von uns im Weiteren entwickelten Typologie liegt eine Mehrebenenuntersuchung zugrunde. Während unsere ursprüngliche Fragestellung auf die spezifischen Potenziale digitaler Medien (Mobiltelefon, E-Mail, Chat, WWW etc.) für eine kommunikative Vernetzung in der Diaspora abhob, machte die Forschung schnell deutlich, dass diese Potenziale nur gefasst werden können, wenn man sie im Gesamtkontext der Aneignung auch traditioneller Massenmedien sieht.

Eine solche Forschung fügt sich in eine Betrachtungsperspektive, die sich mit den „Medienrepertoires“ von Migrantinnen und Migranten in heutigen, sich wandelnden „Medienumgebungen“ auseinandersetzt. Der Begriff der „Medienumgebung“ (Meyrowitz 1995: 50; Krotz 2006: 33) wurde von der Mediumstheorie geprägt und hebt darauf $\mathrm{ab}$, dass Medien in ihrem Gesamtarrangement zu einem bestimmten Zeitpunkt charakteristische „kulturelle Umgebungen“ produzieren. Der Ausdruck des „Medienrepertoires“ (Hasebrink/Popp 2006) fasst konkreter aus Sicht einer einzelnen Person „die Gesamtheit der genutzten Medienangebote (Prinzip der Ganzheitlichkeit) und der wechselseitigen Beziehungen zwischen ihnen [...] (Prinzip der Relationalität)“ (Hasebrink/Domeyer 2010: 51). Entsprechend ist mit den in der Mediennutzungsforschung zunehmend verbreiteten Konzepten der Medienumgebung und des Medienrepertoires ein Blickwinkel verbunden, der versucht, die Nutzung resp. Aneignung von Medien nicht im Hinblick auf Einzelmedien, sondern insgesamt zu erfassen.

Diese integrative, transmediale Orientierung teilen wir in unserer Studie. Sprechen wir im Weiteren also von der kommunikativen Konnektivität oder Vernetzung einer Person bzw. eines Typus von Personen, so begreifen wir darunter die Gesamtheit der sich wechselseitig bedingenden massenmedialen und personalen Kommunikationsbeziehungen in ihrer jeweiligen Spezifik. Diese ergeben sich nicht durch die Aneignung eines einzelnen Mediums (bspw. des Fernsehens), sondern über das Ineinandergreifen der verschiedensten Medien im Gesamtrepertoire einer Person.

Konkret liegt unserer Forschung - neben Sekundärauswertungen bestehender, standardisierter Studien zur Mediennutzung von Migrantinnen und Migranten ${ }^{6}$ - eine medienethnografische Untersuchung mit einem transkulturellen Vergleichsdesign zugrunde. Ein solches Vorgehen liegt darin begründet, dass es uns darum geht, kulturelle Iden-

6 Da diese Sekundäranalysen für die im Weiteren vorgestellte Typenbildung nicht relevant sind, gehen wir nicht näher auf diese ein. Für die Gesamtergebnisse vgl. die sich in Vorbereitung befindende Projektpublikation (Hepp et al. 2011). 
tität und kommunikative Vernetzung aus subjektiver Sicht der Migrantinnen und Migranten verstehend zu rekonstruieren. Das Konzept der Medienethnografie hebt im Gegensatz zur allgemeinen Ethnografie nicht darauf ab, Lebenswelten in deren Gesamtheit "dicht“ (Geertz 1997) zu beschreiben, sondern versteht sich als ein methodischer Ansatz der „Ethnografie über Menschen, die Medien nutzen, konsumieren, distribuieren oder produzieren“ (Bachmann/Wittel 2006: 183). Untersucht wird demnach die kontextualisierte Aneignung von Medien. Aus diesem Grund ist das medienethnografische Vorgehen in den meisten Fällen keine „ethnography proper“ - operiert also nicht mit langmonatigen Feldaufenthalten (Lotz 2000). Genutzt werden dagegen „akkumulierte ethnografische Miniaturen“ (Vielzahl von Kurzaufenthalten, Beobachtungen und Interviews) bzw. eine „virtuelle Ethnografie“ (Hine 2000) oder „Netnografie“ (Kozinets 2010) (ethnografische Verfahren, die sich rein auf den Kommunikationsraum des Internets beziehen), um Aussagen in Bezug auf spezifische Umgangsweisen mit Medien zu machen.

Unsere Untersuchung bewegt sich in einem auf diese Weise verstandenen medienethnografischen Gesamtrahmen und bezieht in diesem eine qualitative Netzwerkanalyse ein (Hollstein 2006). Dabei ist unser medienethnografisches Vorgehen als „Rahmenstrategie zur Generierung von Theorien“ (Krotz 2005: 247) insgesamt auf Theorieentwicklung ausgelegt und operiert mit einer multiperspektivischen, translokal fokussierten Materialerhebung (Marcus 1995). Die untersuchten Migrationsgemeinschaften wurden nach dem Prinzip der Varianz ausgewählt, erstens was die Herkunftsregion betrifft (Länder an verschiedenen Grenzen Europas), zweitens was die Größe betrifft (mit der türkischen und russischen Diaspora zwei größere Migrationsgruppen, mit der marokkanischen eine kleinere) und drittens was die zeitliche Hochphase der Migration betrifft (mit der türkischen eine Gruppe breiter Migration seit den 1970er Jahren, mit den beiden anderen kontinuierlichere bzw. jüngere Migrationsgruppen).

Im Einzelnen haben wir folgendes Material erhoben, auf das sich die weitere Argumentation stützt: Die Daten wurden in der marokkanischen, russischen und türkischen Diasporagemeinschaft in zwei deutschen Großstädten (Berlin und Bremen) und deren Umland je bei 31 bis 37 Personen erhoben. ${ }^{7}$ Diese wurden nach dem Prinzip der Streuung über die Kategorien Alter, Aufenthaltszeit in Deutschland, Bildung und Gender ausgewählt. Kriterien für die Auswahl einzelner Personen war die subjektive Zugehörigkeit zu einer der Migrationsgemeinschaften. ${ }^{8}$ Es wurden von uns qualitative Interviews geführt sowie von den Interviewten offene Netzwerkkarten und - wo möglich zweiwöchige Medientagebücher erstellt. Bei den offenen Netzwerkkarten handelt es sich um freie, während des Interviews näher erläuterte Zeichnungen, die darstellen, wie die interviewten Personen ihr jeweiliges Kommunikationsnetzwerk sehen. Diese dienten in Triangulation mit den Erläuterungen der Person der Erfassung der Strukturen kommunikativer Vernetzung, während es uns die Medientagebücher ermöglichen, über zwei Wochen hinweg die Prozesse kommunikativer Vernetzung zu rekonstruieren. Ergänzt wurden solche Materialien durch weitere materiale Dokumentationen (u. a. Fotografien von privaten und öffentlichen Mediennutzungsorten). Das Gesamtmaterial wurde in einem an die Grounded Theory (Glaser/Strauss 1999; Krotz 2005) angelehnten Prozess

7 Die Gesamtzahl der untersuchten Fälle beträgt 100, 32 Fälle der marokkanischen Diaspora, 31 Fälle der russischen Diaspora und 37 Fälle der türkischen Diaspora.

8 Dieses Kriterien führt bspw. bei der russischen Diaspora dazu, dass hierunter Personen aus verschiedenen Ländern der ehemaligen UdSSR fallen, die Aussiedler, Kontingentflüchtlinge sowie Bildungs- und Wirtschaftsmigranten sind, gemeinsam aber die in unterschiedlichen Graden bestehende subjektive Zugehörigkeit zu der betreffenden Migrationsgemeinschaft teilen. 
des (offenen) Kodierens ausgewertet. ${ }^{9}$ Leitend dafür war im Sinne unserer Forschungsziele die Beschreibung alltagsweltlich kontextualisierter kultureller Muster der Medienaneignung in den drei Diasporagemeinschaften sowie deren kommunikative Vernetzung im Hinblick auf kulturelle Identitätsbildung.

Wenn wir hier von kulturellen Mustern sprechen, so fassen wir damit im Sinne der verstehenden Sozialforschung „regelhafte Formen“. Hierbei stehen „Muster des Denkens“, „Muster des Diskurses“ und „Muster der Praxis“ bzw. des „Handelns“ in Wechselbeziehung zueinander (Hepp 2009). Der Gebrauch des Ausdrucks Muster verweist nicht auf etwas Statisches. Im Gegensatz dazu gehen wir davon aus, dass es auch um Muster des Prozesses geht. Insgesamt hebt der Begriff Muster darauf ab, nicht einfach das singuläre Denken, den singulären Diskurs oder die singuläre Praxis zu beschreiben. Vielmehr geht es darum, auf der Basis der Analyse unterschiedlicher singulärer Phänomene die typischen Arten des Denkens, der Diskurse oder der Praktiken in einem bestimmten kulturellen Kontext herauszuarbeiten. Mit anderen Worten zielt unsere Forschung auf eine qualitative Typenbildung. Unser gesamter Auswertungsprozess ist dabei „transkulturell“ angelegt. Das heißt, wir vermeiden bei der Kategorienbildung, diese von vornherein auf die untersuchten einzelnen Diasporagemeinschaften zu beziehen, sondern erarbeiten ein Kategoriensystem über diese hinweg.

Als Ergebnis dieses Kodierungsprozesses liegt ein System von insgesamt 86 Einzelkategorien vor. Diese lassen sich in den beiden Schlüsselkategorien kulturelle Identität und kommunikative Vernetzung bzw. elf weiteren Subkategorien systematisieren. So fassen wir kulturelle Identität über die Subkategorien Angaben zur Person, Identitätsbild, Migrationserleben und Wohnorte, worüber dann die identitätsbezogenen Einzelkategorien systematisiert sind. Kommunikative Vernetzung verweist auf die Subkategorien Angebotsformen, Medienausstattung, Kommunikationsnetzwerk, Inbalte, Nutzungsorte, Medienerfahrung und Vermittlerrollen, die wiederum weitere Einzelkategorien erschließen.

Im Sinne der qualitativen Sozialforschung ist ein solches Vorgehen der Grounded Theory zirkulär: Einerseits gehen wir von an der bestehenden Forschung orientierten Begriffen von kultureller Identität und kommunikativer Vernetzung aus. Während wir unter kommunikativer Vernetzung wie bereits formuliert die Gesamtheit der sich wechselseitig bedingenden massenmedialen und personalen Kommunikationsbeziehungen fassen, bezeichnen wir mit kultureller Identität die Artikulation von „instabile[n] Identifikationspunkte[n]“ (Hall 1994: 30), d. h. die fortlaufende differenz- und damit sinnstiftende Selbstpositionierung einer Person, insbesondere im Hinblick auf damit einhergehende kulturelle Zugehörigkeiten und Vergemeinschaftungen. Andererseits ermöglicht es erst unsere Musteranalyse, beide zunächst einmal offenen Grundverständnisse von kommunikativer Vernetzung und kultureller Identität als Schlüsselkategorien der Typenbildung zu benennen und im Hinblick auf das Untersuchungsfeld zu füllen. Insgesamt bezeichnen wir den damit typologisierten Zusammenhang als Aneignungstypen um zu verdeutlichen, dass sich unsere Typologie als materialbasierte Theorie auf das Untersuchungsfeld der Medienaneignung bezieht.

9 Die Kodierung erfolgte mittels des Programms Hyperresearch 2.6. 


\section{Aneignungstypen kultureller Identität und kommunikativer Vernetzung: Herkunfts-, Ethno- und Weltorientierte}

In Kombination unserer beiden Hauptkategorien - kulturelle Identität und kommunikative Vernetzung - lassen sich über die von uns untersuchten Diasporagemeinschaften hinweg drei Medienaneignungstypen unterscheiden, nämlich „Herkunftsorientierte“ („origin oriented“), „Ethnoorientierte“ („ethno oriented“) und „Weltorientierte“ („world oriented“) (siehe Abbildung 1). Während diese drei Typen für jede der drei Diasporagemeinschaften eine spezifische Ausprägung haben bzw. bezogen auf die Diasporas ungleich verteilt sind, bieten sie insgesamt die Möglichkeit, das Potenzial von Medien für Migrantinnen und Migranten abzuschätzen.

\section{Abbildung 1: Aneignungstypen}

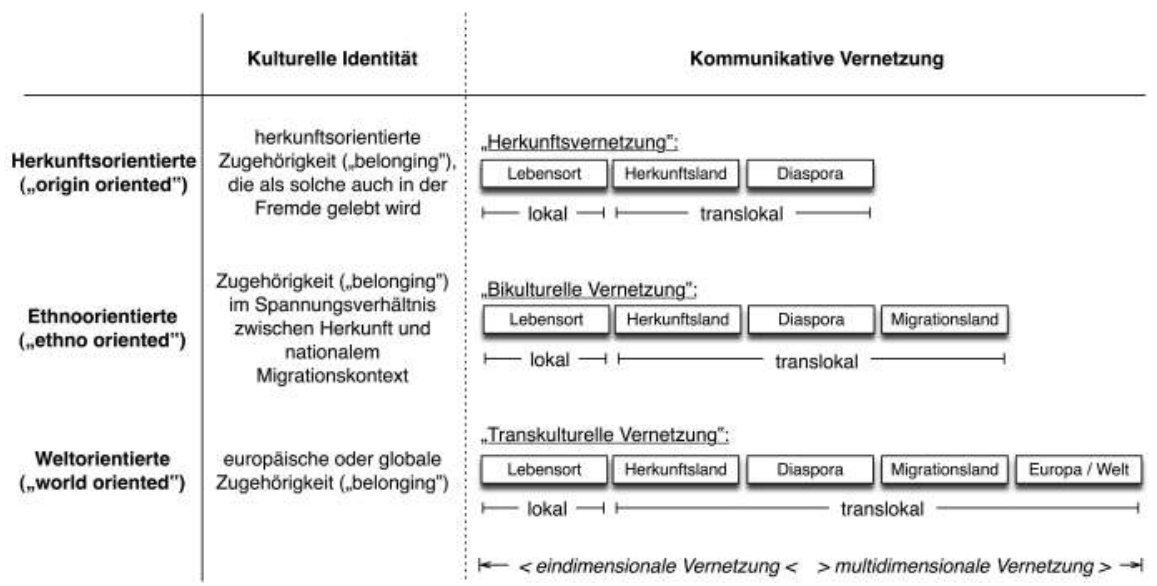

Die Namen der drei von uns unterschiedenen Typen sind zuerst einmal anhand der subjektiven Positionierung der eigenen kulturellen Identität gebildet, d. h. auf Basis der Selbstzuschreibungen der von uns interviewten Migrantinnen und Migranten in den erhobenen Interviews (systematisiert entlang der weiter differenzierenden Subkategorien Angaben zur Person, Identitätsbild, Migrationserleben und Wobnorte). Hiermit korrespondiert tendenziell die kommunikative Vernetzung, die wir aus den Interviewangaben zur Medienaneignung und aus den Netzwerkkarten bestimmt haben (entlang der Subkategorien Angebotsformen, Kommunikationsformen, Medienausstattung, Kommunikationsnetzwerk, Inhalte, Nutzungsorte, Medienerfahrung und Vermittlerrollen). Dabei kann die oben stehende Abbildung nur unzureichend fassen, dass sich über die Typen hinweg auch die Qualität der kommunikativen Vernetzung ändert. Damit meinen wir, dass sich die Sinndimension dessen, was die kommunikative Vernetzung am Lebensort, zum Herkunftsland, zur Diaspora etc. ausmacht, von Typ zu Typ ändert. Zentral dabei ist die Unterscheidung zwischen der lokalen und translokalen kommunikativen Vernetzung. Mit lokaler kommunikativer Vernetzung bezeichnen wir die Vernetzung am aktuellen Lebensort, d. h. dem direkten, alltagsweltlichen Lebensumfeld, die - neben Face-to-Face-Kommunikation - durch Medien der personalen Kommunikation (Mobiltelefon, E-Mail etc.) wie auch durch Medien der Massenkommunikation (Tageszeitung, Lokalradio etc.) geschieht. Unter translokaler kommunikativer Vernetzung ver- 
stehen wir die ortsübergreifende kommunikative Vernetzung, für die - neben Reisen ${ }^{10}$ - primär die verschiedenen Medien relevant sind. Die translokale kommunikative Vernetzung kann auf das Herkunftsland, die Diaspora-Gemeinschaft, das Migrationsland oder weitergehende Sozialräume (bspw. Europa) ausgerichtet sein.

Vereinfacht formuliert lässt sich sagen, dass Herkunftsorientierte eine subjektiv gefühlte Zugehörigkeit zu ihrer Herkunftsregion haben, die ihr Leben in der „Fremde“ prägt. Diese subjektiv gefühlte Zugehörigkeit kann, muss jedoch nicht auf einer Sozialisation in der Herkunftsregion beruhen. Gerade bei jüngeren Migrantinnen und Migranten, die weitgehend in Deutschland, dort aber stark fokussiert auf die Migrationsgemeinschaft aufgewachsen sind, basiert die herkunftsorientierte Zugehörigkeit durchweg auf Vorstellungen bzw. den Erfahrungen bei einzelnen Urlaubsreisen. Ihre kulturelle Identität charakterisieren die von uns Interviewten dann bspw. als „Marokkaner [...] egal wo ich [...] lebe“ (Fatih, m, 28 Jahre, marokkanische Diaspora), ${ }^{11}$ als die „der sowjetischen [sic!] Kultur" (Pawel, m, 59 Jahre, russische Diaspora) oder schlicht als „Türke“ (Feraye, w, 35 Jahre, türkische Diaspora). Für Herkunftsorientierte ist das Leben in der Fremde nicht unbedingt problematisch. Schwierigkeiten entstehen vor allem dann, wenn sie das subjektive Gefühl haben, mit ihrer eigenen Identität nicht akzeptiert zu sein bzw. in schwierigen ökonomischen Verhältnissen leben.

Diese Orientierung von kultureller Identität geht einher mit einer spezifischen kommunikativen Vernetzung, die sich als Herkunftsvernetzung bezeichnen lässt. Während eine intensive lokale kommunikative Konnektivität am Lebensort besteht, zumeist mit Mitgliedern der eigenen Diasporagemeinschaft, existieren darüber hinaus umfassende translokale Kommunikationsbeziehungen insbesondere zur Herkunftsregion. Das Medienrepertoire dieses Typus ist auf eine solche kommunikative Herkunftsvernetzung orientiert. Beispielsweise hält Noureddin (m, 27 Jahre, marokkanische Diaspora) über Telefon Kontakt zu seiner Verwandtschaft in seinem Herkunftsland, seinen Geschwistern, seiner Großmutter, seinem Onkel und seinen Eltern. Daneben ist das personale Kommunikationsnetzwerk von Herkunftsorientierten vor allem auf im lokalen Umfeld lebende Migranten gleicher Herkunft ausgerichtet. Und auch für Massenmedien ist festzuhalten, dass Herkunftsorientierte tendenziell Herkunftsangebote nutzen bzw. allenfalls das deutsche Fernsehen eine kommunikative Einbettung am aktuellen Lebensort sicherstellt. In ihrer Gesamtheit besteht die Medienausstattung der Herkunftsorientierten je nach Alter, Bildung und ökonomischen Verhältnissen aus unterschiedlichen Medien, die aber insbesondere für eine kommunikative Vernetzung zur Herkunft geeignet sind.

Anders verhält es sich bei dem Ethnoorientierten. Die Bezeichnung dieses Typus hebt darauf ab, dass dieser seine Zugehörigkeit im Spannungsverhältnis zwischen Herkunft und nationalem Aufnahmekontext sieht, er sich also charakteristischerweise Deutschmarokkaner, Deutschtürke oder Deutschrusse nennt. Im Zentrum der Zugehörigkeit steht der Teil der jeweiligen Diasporagemeinschaft, der sich im deutschen Kontext konkretisiert. Mit der Bezeichnung „Ethnoorientierter“ wollen wir verdeutlichen, dass für Migrantinnen und Migranten dieses Typs Fragen der ethnischen Verortung der zentrale Aspekt der Entwicklung von kultureller Identität sind. Mahmut (m, 30 Jahre, türkische Diaspora) meint, er empfände „genauso Türkei als [s]eine Heimat wie Deutschland“, Amir (m, 57 Jahre, marokkanische Diaspora) charakterisiert sich als „Mischling [...] von

10 Der Stellenwert der Verbreitung von Billigflügen für den Zusammenhalt von Migrationsgemeinschaften kann an dieser Stelle nicht weiter vertieft werden.

11 Bei allen Namen handelt es sich um Pseudonyme. Die Zitate wurden geringfügig der deutschen Orthografie und Grammatik angepasst, um die Lesbarkeit zu erleichtern. 
beiden Kulturen“ und Valerij (m, 68 Jahre, russische Diaspora) sagt, „ich kann mich zu der deutschen Kultur nicht zählen alleine“. Ob dieses Spannungsverhältnis von Zugehörigkeit als produktiv, d. h. als eine Chance, oder als entwurzelnd, d. h. als ein Problem, erlebt wird, hängt von unterschiedlichen Faktoren wie Sprachkompetenz, Bildung und ökonomischem Status bzw. der Berufssituation ab. ${ }^{12}$ Als Charakteristikum besteht in jedem Fall eine ethnische Orientierung auf das Spannungsverhältnis von Herkunfts- und Migrationsland.

Die kommunikative Vernetzung des Ethnoorientierten lässt sich als bikulturelle Vernetzung beschreiben. Diese Bezeichnung akzentuiert, dass die kommunikative Vernetzung des Typus in dem Sinne bikulturell ist, dass sie lokal wie translokal vor allem im Spannungsverhältnis zwischen zwei (vorgestellten) Kulturen erfolgt. In diesem Schnittfeld konstituiert sich die Diaspora als eine eigenständige kulturelle Figuration, wobei die Vernetzung in dieser in der Tendenz insofern „national“ gerahmt bleibt, als sie insbesondere auf die Migrationsgemeinschaft in Deutschland gerichtet ist. Gewisse Öffnungen finden vor allem am Lebensort statt: In das lokale Kommunikationsnetzwerk sind Mitglieder der eigenen Diaspora sowie andere Migrantinnen und Migranten bzw. Deutsche einbezogen. Exemplarisch sei Viktoria (w, 47 Jahre, russische Diaspora) zitiert, die als Teil ihres Bekanntenkreises „nicht nur Leute aus Russland“ aufzählt, sondern ebenso „sehr nette Mädchen, die aus der Türkei gekommen sind. Lusie aus Brasilien [...], Evan aus Moldawien [...] er hat mehrere Jahre in Italien gelebt, deswegen weiß ich nicht, wo er herkommt".

Wesentlich vielschichtiger und umfassender als beim Herkunftsorientierten ist auch die translokale kommunikative Vernetzung. Es bestehen nicht nur Kommunikationsbeziehungen zur (vorgestellten) Herkunft. Insbesondere ist eine intensive kommunikative Vernetzung zur eigenen Diaspora bzw. zu Deutschen auszumachen. Diese kommunikative Vernetzung wird im Medienrepertoire der Ethnoorientierten sowohl von (digitalen) Medien der personalen Kommunikation als auch der Massenkommunikation getragen. Dominierende Angebotsformen sind außer lokalen diasporische, deutsche und Herkunfts-Angebote. In diesem Sinne formuliert Aysen (w, 44 Jahre, türkische Diaspora) in Bezug auf ihr Lektüre-Repertoire: „wir kaufen täglich eine türkische und eine deutsche Zeitung, damit wir wissen, was passiert in der Türkei und was passiert hier in Deutschland und hier in Berlin“. Dem entspricht wiederum das translokale personale Kommunikationsnetzwerk des Ethnoorientierten, dessen Reichweite Deutschland und die eigene Herkunft umfasst bzw. neben der Familie und Freunden die Diaspora in Deutschland und Deutsche einbezieht.

Eine nochmals andere kulturelle Identität und kommunikative Vernetzung hat der Weltorientierte. Die Bezeichnung dieses Typus ist unseren Interviews entlehnt, in denen sich von uns Befragte als „Weltmensch“ (Gökce, w, 33 Jahre, türkische Diaspora) oder als „Europäer“ (Danil, m, 24 Jahre, russische Diaspora) bezeichnen. Damit rücken sie Formen von Identität ins Zentrum, die jenseits des Ethnisch-Nationalen (ob in Bezug zur Herkunft oder zum aktuellen Lebenskontext) liegen. Der Begriff des Weltorientierten hebt demnach darauf ab, dass die subjektiv gefühlte kulturelle Zugehörigkeit, auf welchem Niveau auch immer, jenseits des Nationalen liegt. Vorstellungen der Nation ob der deutschen, der Herkunft oder eines bilateralen Spannungsverhältnisses zwischen

12 Entlang dieser Form der subjektiven Wabrnebmung der Ethnoorientierung als „produktiv“ oder „entwurzelnd“ lässt sich unsere Typologie für einzelne Untersuchungsfelder weiter ausdifferenzieren. Exemplarisch dafür kann die Typologie von Düvel (2010) angesehen werden, die sich auf das Untersuchungsfeld von jugendlichen Migrantinnen und Migranten aus Russland bezieht. 
beiden - werden durchschritten und das supranationale Europa oder gar das Menschsein als solches werden zum Bezugspunkt von Zugehörigkeit.

Die subjektiv gefühlte Zugehörigkeit geht mit einer spezifischen kommunikativen Vernetzung einher, die sich als transkulturelle Vernetzung titulieren lässt. Diese Benennung macht deutlich, dass der Einschluss von Herkunfts- und Migrationskontext in der kommunikativen Vernetzung durchaus mit dem Typus des Ethnoorientierten zu vergleichen ist. In Differenz zu diesem ist die Reichweite kommunikativer Vernetzung aber umfassender und tendiert zum Europäischen oder (vorgestellten) Globalen bzw. konkreter zu einer Erstreckung des kommunikativen Netzwerks über verschiedenste Länder und Kulturen hinweg. Das Medienrepertoire ist sehr breit angelegt. Außer unterschiedlichen Massenmedien spielen insbesondere Medien der personalen Kommunikation und unter diesen digitale Medien eine große Rolle. „Weltorientierte“ nutzen neben E-Mail, Telefon und Chat zum Teil in hoher Intensität Social-Web-Angebote - also sogenannte Web 2.0-Anwendungen wie Facebook -, um mit den Personen ihres Netzwerks in Beziehung zu bleiben. In diesem Netzwerk sind Familien- und Diaspora-Angehörige ein wichtiger Bezug. Daneben umfasst das Kommunikationsnetzwerk eine Vielzahl weiterer Personen, zu denen der Kontakt zum Teil über Beruf und Ausbildung, zum Teil über private Anlässe aufgebaut wurde und mehr oder weniger intensiv gepflegt wird. Dabei ist die Migrationserfahrung durchaus ein Potenzial der Kontaktentwicklung. Um hier exemplarisch für andere Inaya (w, 29 Jahre, marokkanische Diaspora) zu zitieren:

„I have two cousins in Paris - oh no, one in Paris, one in Saint-Etienne, in France - and one cousin in Moscow and I have so many friends abroad because we studied together in Morocco and all of us went out to finish our postgraduate studies. So there are many in France mainly and in Canada because in the Quebec part, in the French speaking part of Canada.“

Wir können also festhalten, dass für jeden der drei Grundtypen eine je spezifische wechselseitige Beziehung von kultureller Identität und kommunikativer Vernetzung besteht, die sich nicht in einseitige Kausalitäten auflösen lässt. Das heißt, eine herkunftsorientierte, ethnoorientierte oder weltorientierte kulturelle Identität resp. subjektive Zugehörigkeit hat nicht eine bestimmte kommunikative Vernetzung zur Folge. Ebenso zieht eine Herkunftsvernetzung, eine bikulturelle Vernetzung oder eine transkulturelle Vernetzung keine spezifische kulturelle Identität nach sich. Vielmehr ist die Beziehung zwischen beiden so zu sehen, dass sich eine bestimmte kommunikative Vernetzung und eine bestimmte Form kultureller Identität zusammen artikulieren. Man muss von einer wechselseitigen Verstärkung beider Aspekte ausgehen: Die Herkunftsvernetzung verstärkt die Artikulation einer herkunftsorientierten kulturellen Identität und damit wiederum eine Ausrichtung auf ein entsprechendes Kommunikationsnetzwerk. Eine bikulturelle Vernetzung verstärkt die Artikulation einer doppelten kulturellen Zugehörigkeit und damit wiederum eine Orientierung auf ein Kommunikationsnetzwerk zwischen Herkunft und Migrationsland. Eine transkulturelle Vernetzung verstärkt die Artikulation einer europäischen bzw. globalen Zugehörigkeit und so eine Fokussierung auf ein weitreichendes transnationales Kommunikationsnetzwerk. Bei all diesen Prozessen greifen Medien der personalen Kommunikation (Telefon, Mobiltelefon, E-Mail, Social Web) und Medien der Massenkommunikation (Fernsehen, Zeitung, WWW) umfassend ineinander. 


\section{Konkretisierungen: Typenausprägungen über die Migrationsgemeinschaften hinweg}

Die Zitate, mit denen wir unsere Analyse belegt haben, machen bereits deutlich, dass die von uns unterschiedenen Typen nicht gleich über die Migrationsgemeinschaften verteilt sind bzw. ausgehend von einer solchen Typologie die Notwendigkeit einer differenzierten Betrachtung der einzelnen Fälle besteht. Ganz in diesem Sinne begreifen wir die von uns entwickelte Typologie nicht als Unterfangen, die untersuchten Migrationsgemeinschaften zu homogenisieren. Vielmehr sind diese in sich ähnlich vielfältige Figurationen, wie es territoriale Vergemeinschaftungen der Nation sind (Robins/Aksoy 2006). Entsprechend ist die Typologie als Ansatzpunkt zu verstehen, um die Spezifik der Medienaneignung sowohl einzelner Diasporas als auch einzelner Untergruppen in diesen vergleichend zu erfassen. Während es den Rahmen dieses Aufsatzes sprengen würde, dies für alle Medienaneignungsformen im Detail zu diskutieren (siehe Hepp et al. 2011), wollen wir einige Grundmomente solcher Spezifika anhand der Typenausprägungen über die Migrationsgemeinschaften hinweg deutlich machen.

Die von uns insgesamt erforschten 100 Fälle ermöglichen keine statistisch repräsentativen Aussagen. Da die Fallauswahl gemäß des Verfahrens des theoretischen Samplings aber den Charakter der jeweiligen Migrationsgemeinschaft erfasst und wir davon ausgehen, in unserem Forschungsprozess eine theoretische Sättigung erreicht zu haben, halten wir doch eine Verallgemeinerbarkeit der folgenden Überlegungen als Tendenzaussagen für die untersuchten Diasporas für möglich.

\section{Tabelle 1: Typen nach Diaspora (absolute Zablen)}

\begin{tabular}{lccc}
\hline & Herkunftsorientierte & Ethnoorientierte & Weltorientierte \\
\hline $\begin{array}{l}\text { Marokkanische Diaspora } \\
\text { (n=32) }\end{array}$ & 9 & 18 & 5 \\
Russische Diaspora $(\mathrm{n}=31)$ & 9 & 17 & 5 \\
Türkische Diaspora $(\mathrm{n}=37)$ & 12 & 23 & 2 \\
\hline Gesamt & 30 & 58 & 12 \\
\hline
\end{tabular}

Betrachtet man als Erstes die Verteilung der von uns unterschiedenen Typen über die Migrationsgemeinschaften hinweg (Tabelle 1), so zeigt sich, dass der Typus des Ethnoorientierten klar dominiert, gefolgt vom Herkunftsorientierten. Was die Fallzahl betrifft, so treten Weltorientierte deutlich seltener auf, insbesondere in der türkischen Diaspora.

Ebenso lassen sich in Bezug auf das Alter Unterschiede für die Typen und ihre Verteilung über die Migrationsgemeinschaften ausmachen (Abbildung 2). Man kann für Herkunftsorientierte vor allem in der türkischen Diaspora sagen, dass diese tendenziell älter sind als die Ethnoorientierten. Dies steht letztlich dafür, dass die türkische Diaspora in Deutschland im Vergleich zu den beiden anderen von uns untersuchten Migrationsgemeinschaften die seit dem Zweiten Weltkrieg am weitesten zurückreichende Einwanderungstradition hat. Auffallend ist das Alter der Weltorientierten: Diese sind die mit Abstand Jüngsten. ${ }^{13}$

13 Hierbei ist im Blick zu haben, dass die Migranten in Deutschland im Durchschnitt zehn Jahre jünger sind als die deutsche Bevölkerung (vgl. Simon 2007: 429), was sich ebenfalls im Durchschnittsalter der von uns interviewten Migranten manifestiert. 


\section{Abbildung 2: Altersverteilung der Aneignungstypen nach Diaspora (Mittelwert)}

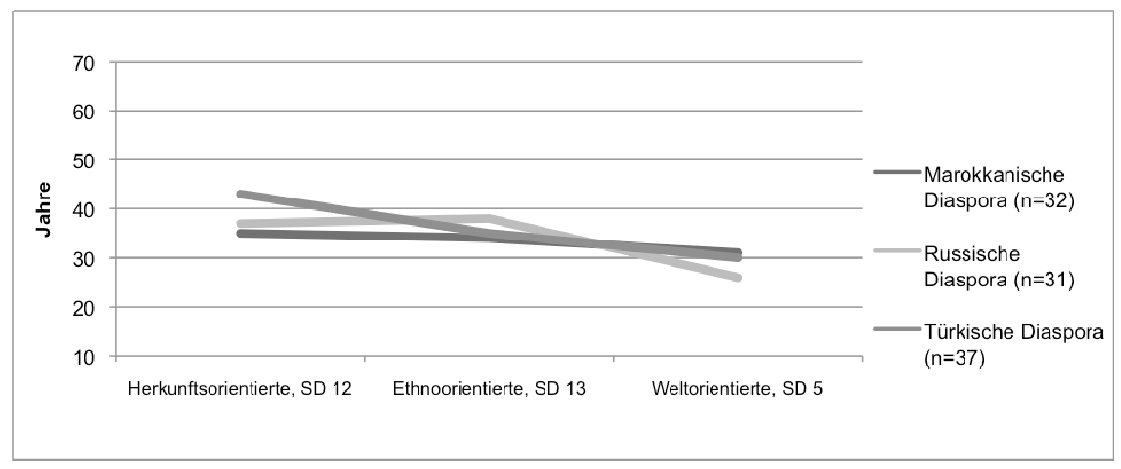

Betrachtet man die von uns interviewten Migrantinnen und Migranten nach ihrem Bildungsniveau, ${ }^{14}$ so ist festzuhalten, dass die formale Bildung nicht der aktuellen Berufstätigkeit entsprechen muss. Es ist deren wiederholte Erfahrung, dass Bildungsabschlüsse aus dem Ausland in Deutschland nicht anerkannt werden und sie folglich in für ihre Ausbildung nicht adäquaten Tätigkeitsfeldern arbeiten. Hat man diese Einschränkung im Blick, fällt doch auf, dass allein schon das Formalkriterium des Bildungsabschlusses eine einfache Stereotypisierung unmöglich macht. Unter Herkunftsorientierten finden sich in allen Migrationsgruppen Personen mit sehr unterschiedlichem Formalabschluss. Bezieht man in die Betrachtung den Umstand mit ein, dass die von uns interviewten Weltorientierten ohne Schulabschluss zumeist noch in der Schule sind bzw. viele derjenigen ohne Hochschulabschluss noch studieren, so lässt sich umgekehrt allerdings argumentieren, dass bei den Weltorientierten eine Tendenz zu höherer Bildung besteht. Eine solche vielfache Differenzierung der einzelnen Typen wird daneben deutlich, wenn wir die Frage der Sprachkompetenz differenzierter betrachten. ${ }^{15}$

Die Abbildung 4 macht zuerst einmal deutlich, dass das Stereotyp, Herkunftsorientierte könnten generell kein Deutsch, nicht trägt. Gleichwohl sind über alle Migrationsgemeinschaften hinweg die Deutschkenntnisse der Herkunftsorientierten am schlechtesten. Auch hier muss man aber vorsichtig sein mit einem verkürzenden Bild: So sind die Kenntnisse der Herkunftssprache gerade bei den Herkunftsorientierten auf einem hohen Niveau, wohingegen Ethnoorientierte - insbesondere was die schriftsprachliche Kompetenz betrifft - in der Herkunftssprache Schwierigkeiten haben, was zum Teil ihre

14 Obige Darstellung ist in dem Sinne konservativ, dass als deren Basis der vorliegende Abschluss zählt, d. h. bspw. Personen in Berufsausbildung werden als Personen mit Schul-, aber ohne Berufsabschluss gewertet.

15 Indikatoren für die Einschätzung der Sprachkenntnisse sind einerseits die Selbsteinschätzung der Interviewten, andererseits unsere Beobachtungen im Interview. „Sehr gute bis gute“ Deutschkenntnisse ist dabei definiert als die Fähigkeit, problemlos alle Fragen im Interview zu verstehen und entsprechend zu antworten, in Kombination mit eigenen Auskünften zu sehr guten bis guten schriftlichen Fähigkeiten. Als „zufriedenstellende“ Deutschkenntnisse charakterisieren wir das Verstehen der Interviewfragen bei einzelnen Problemen mit einer korrekten Antwort in Kombination mit der Selbsteinschätzung nicht hinreichender schriftlicher Kompetenzen. „Geringe“ Deutschkenntnisse konstatieren wir dann, wenn ein Gespräch auf Deutsch wegen mangelnden Verständnis- und Sprechvermögens fast unmöglich war und zumeist in eine andere Sprache gewechselt werden musste (Herkunftssprache oder Englisch). 
Abbildung 3: Bildungsniveau nach Aneignungstyp und Diaspora
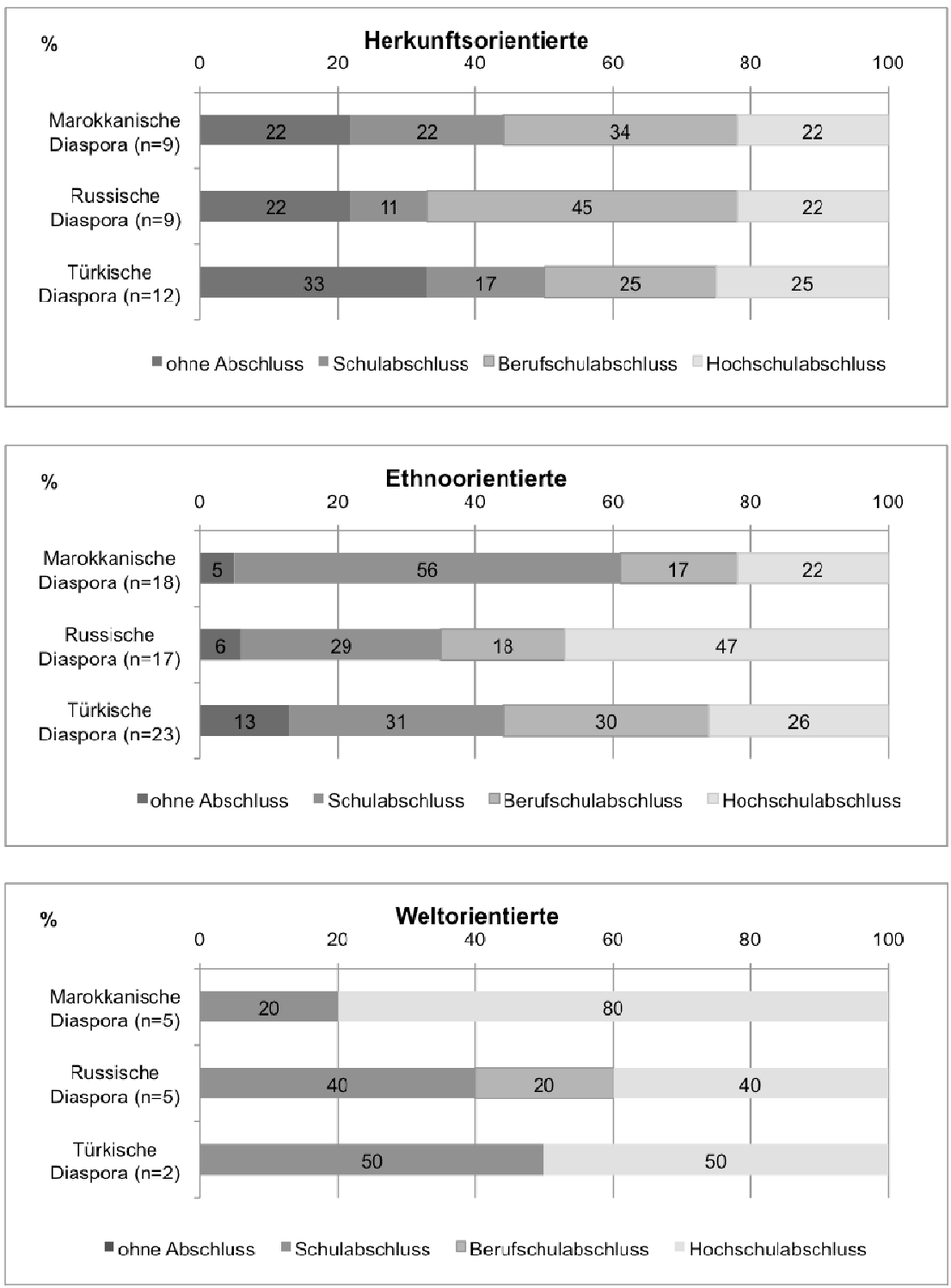

Medienaneignung (bspw. beim Chatten in der Herkunftssprache) einschränkt. Dem gegenüber kommt bei den Weltorientierten hinzu, dass sie über zum Teil sehr gute Kenntnisse einer weiteren Sprache jenseits von Herkunfts- und Migrationssprache verfügen. 
Abbildung 4: Deutschkenntnisse nach Aneignungstyp und Diaspora
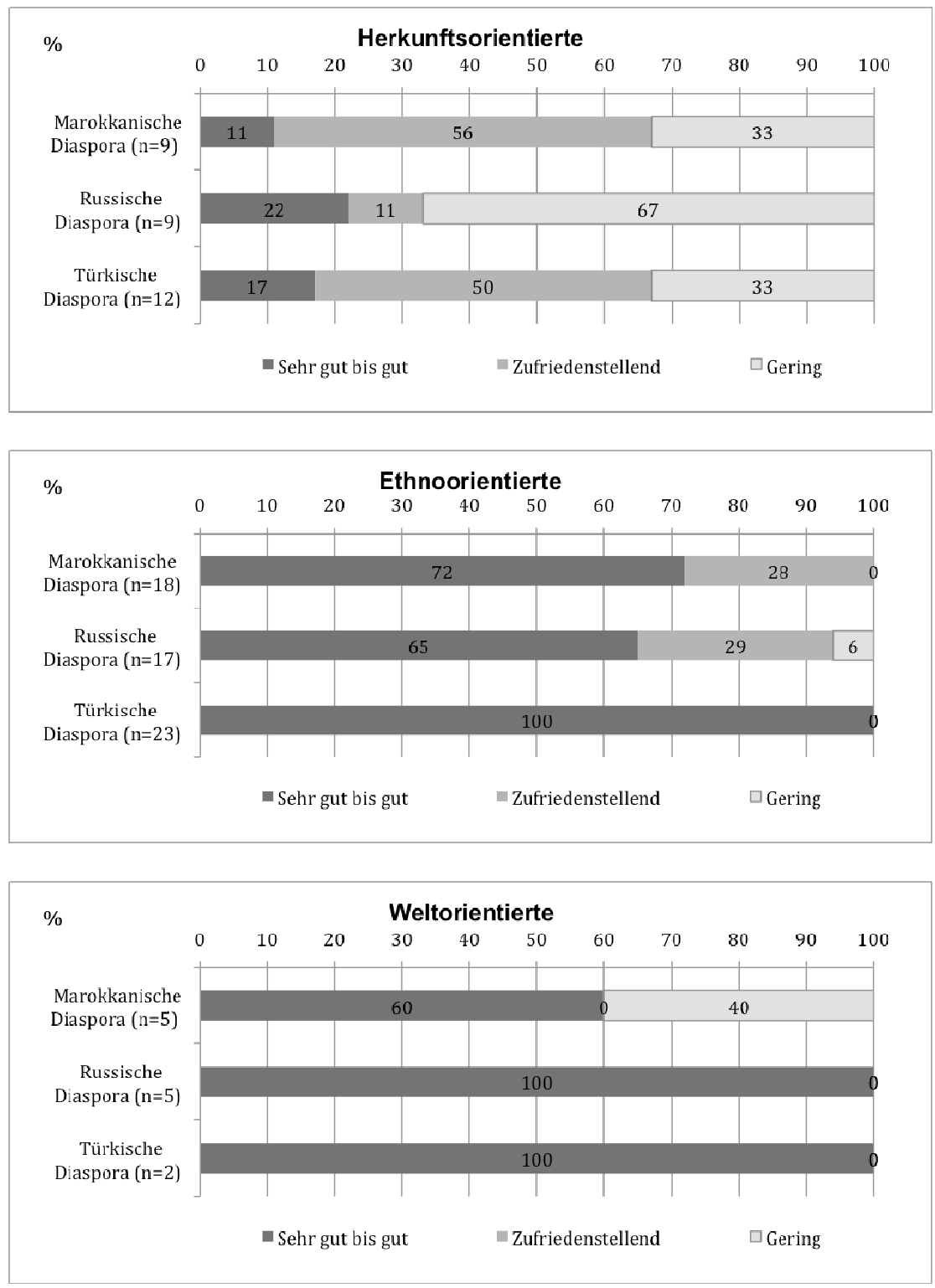
Abbildung 5: Kommunikationsnetzwerk und Sprache

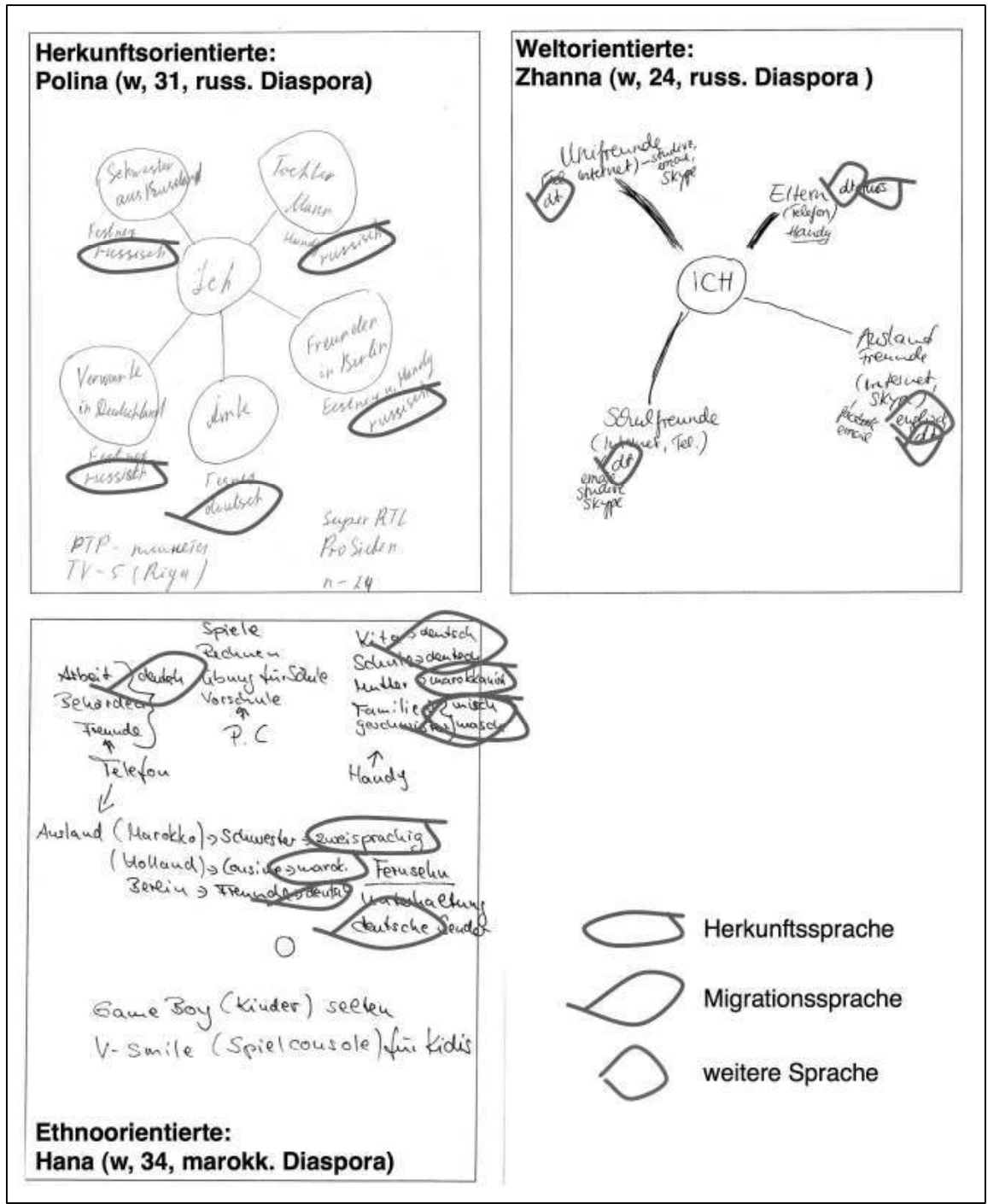

Dies ist zumeist Englisch, in der marokkanischen Diaspora aber auch Französisch, Hocharabisch oder Spanisch. ${ }^{16}$

16 Für die marokkanische Diaspora ist dabei anzumerken, dass für deren Mitglieder Hocharabisch dann eine (bedingte) Fremdsprache darstellen kann, wenn deren Muttersprache Berberisch ist. Daneben ist darauf hinzuweisen, dass auch Herkunfts- und Ethnoorientierte der marokkanischen Diaspora über gute bis sehr gute Kenntnisse weiterer Fremdsprachen verfügen. 
Sehr deutlich werden diese Zusammenhänge, wenn man an dieser Stelle nochmals unser qualitatives Material aufgreift, in diesem Fall nicht die Interviews, sondern exemplarische Netzwerkkarten (siehe Abbildung 5). Ein erstes Beispiel ist die herkunftsorientierte Polina (russische Diaspora). Deutlich zeigt die Netzwerkkarte ihre Herkunftsund (lokale) Diasporavernetzung Face-to-Face und mittels unterschiedlicher Medien. Deutsch wird primär bei institutionalisierten Kommunikationskontakten (bspw. Ärzte, Behörden) oder passiv beim Fernsehen genutzt, ansonsten dominiert klar Russisch. Die bikulturelle Vernetzung der Ethnoorientierten Hana (marokkanische Diaspora) findet mittels Herkunftssprache wie auch der Sprache des Migrationslandes statt bzw. als - wie es die Interviewte selbst bezeichnet - „Mischmasch“ beider Sprachen, insbesondere bei der personalen Kommunikation in der Familie. Die Weltorientierte Zhanna (russische Diaspora) ist - wie die freie Netzwerkkarte zeigt - breit und mittels unterschiedlichster Medien kommunikativ vernetzt. Im Hinblick auf Sprache manifestiert sich in der Netzwerkkarte, inwieweit bei der Vernetzung mittels personaler Kommunikation ins Ausland neben der Herkunftssprache und der Sprache des Migrationslands Englisch einen Stellenwert hat. Ethnoorientierte, vor allem aber Weltorientierte wissen dabei das Potenzial ihrer Mehrsprachigkeit im Einzelfall durchaus zu nutzen, wenn sie sich bspw. darüber Berufsfelder eröffnen. Aber auch die Sprachkompetenz einer Herkunftsorientierung kann Potenziale bieten, bspw. wenn sich durch sie zusammen mit einer technischen Kompetenz das Berufsfeld einer herkunftssprachlichen Konfiguration von Computern eröffnet.

Entsprechend können wir festhalten, dass im Hinblick auf die Ausprägung der von uns unterschiedenen Typen über die verschiedenen Diasporas hinweg zwar bestimmte Tendenzen bestehen. Vor allem die Weltorientierten sind tendenziell sprachkompetenter, gebildeter und jünger als andere Migrantinnen und Migranten bzw. finden sich seltener in der türkischen Diaspora als in den beiden weiteren Migrationsgemeinschaften. Eine einfache Stereotypisierung der drei Typen ist aber unmöglich. So sind alle drei in ihrem Verhältnis zueinander - mit Ausnahme der Weltorientierten in der türkischen Migrationsgemeinschaft - nahezu gleich verteilt über die einzelnen Diasporas. Und auch Menschen mit hoher Bildung und guten Deutschkenntnissen können sehr wohl im Hinblick auf ihre Medienaneignung heimatorientiert sein.

\section{Fazit: Die Vielschichtigkeit kommunikativer Integration}

Ausgangspunkt unseres Artikels war eine Kritik an bestehenden Typologien der Mediennutzung von Migrantinnen und Migranten im Hinblick darauf, dass diese vorschnell die typisierende Beschreibung migrantischer Mediennutzung und -aneignung auf Fragen der Integration reduzieren. Dem haben wir ein offeneres Vorgehen einer typologisierenden Betrachtung der Medienaneignung gegenübergestellt, das bei einer Gesamtbetrachtung des Wechselverhältnisses von kultureller Identität und kommunikativer Vernetzung ansetzt. Hierüber haben wir drei Aneignungstypen unterschieden, Herkunfts-, Ethno- und Weltorientierte, und in Bezug auf diese Typen argumentiert, dass kulturelle Identität und kommunikative Vernetzung ko-artikuliert werden. Eine weitergehende Betrachtung der Typen im Hinblick auf deren Ausprägung über die verschiedenen Migrationsgemeinschaften hinweg hat gezeigt, dass eine stereotype Charakterisierung eines Typus als „ungebildet“, „alt“ oder „sprachlich inkompetent“ verkürzend ist, sondern wir es mit vielschichtigeren Zusammenhängen der Sinnproduktion in einem Leben in der Diaspora zu tun haben.

Bewertet man nun abschließend eine solche Typologie im Hinblick auf die wissenschaftliche Diskussion um „Medien und Integration“, so macht sie unseres Erachtens 
die Notwendigkeit eines komplexeren Bewertungsrahmens deutlich, als er bisherigen Nutzungstypologien zugrunde lag. Greifen wir hier nochmals den bereits angeführten kommunikations- und medienwissenschaftlichen Integrationsbegriff auf, wonach Integration „der erwünschte soziale Prozess [ist], der die Teile einer Gesellschaft (Individuen, Institutionen, Gruppen) unter Mitwirkung ihres Bewusstseins mehr oder weniger stark zum Ganzen dieser Gesellschaft verbindet“ (Pöttker 2005: 40f.), ein Prozess, der auch über (Medien-)Kommunikation vermittelt ist. Entsprechend werden - wie bereits formuliert - als einer der Indikatoren von Integration „Stärke und Inhalt der Kommunikation zwischen Teilen einer Gesellschaft“ (ebd.) begriffen. Unsere Analysen machen deutlich, dass Integration und (Medien-)Kommunikation - aus der Perspektive der Migrantinnen und Migranten rekonstruiert - die Schwierigkeit einer solchen Definition deutlich macht, indem die Referenz „des Erwünschten“ (von wem erwünscht?) wie „der Gesellschaft“ (die Gesellschaft der Herkunft, Migration oder Diaspora?) durchaus vielfältig sein kann.

Zugespitzt formuliert kann man ausgehend von unserer Analyse Folgendes festhalten: Für Herkunftsorientierte ist eher eine eindimensionale kommunikative Vernetzung kennzeichnend, also der Aufbau von lokalen wie herkunftsorientierten Kommunikationsnetzwerken. Dies heißt aber nicht zwangsläufig, dass sie in dem von der Forschung zu Medien und Migration gerne unterstellten Rahmen „kommunikativ desintegriert“ wären: Die kommunikative Integration der Herkunftsorientierten in ihre Migrationsgemeinschaft ist mitunter in hohem Maße intensiv, aber eben herkunftsorientiert. Ethnoorientierte sind gemeinhin im Hinblick auf die jeweiligen Bezugsgruppen multidimensionaler vernetzt, was im positiven Falle eine „kommunikative Integration“ sowohl im Hinblick auf das Migrationsland als auch die Diaspora gestattet, im negativen Fall jedoch mit der Erfahrung einer Verunsicherung im Hinblick auf die eigene Zughörigkeit einhergeht. Die weitreichende Multidimensionalität der kommunikativen Vernetzung von Weltorientierten korrespondiert damit, dass sich deren „kommunikative Integration" vom rein Nationalen zu supranationalen Einheiten wie beispielsweise Europa verlagert bzw. sich gänzlich von einer einfachen Staatsbezüglichkeit lösen kann.

Solche Forschungsergebnisse machen nicht nur deutlich, dass wir uns von der Betrachtung von Einzelmedien lösen müssen, wenn wir uns dem Stellenwert von Medien für Migrantinnen und Migranten annähern wollen. Darüber hinaus wird greifbar, dass wir es mit einer mehrdimensionalen kommunikativen Vernetzung zu tun haben, der eine entsprechende Vielfalt von „kommunikativen Integrationsbezügen“ entspricht. Betrachtet man diese Ergebnisse wertend, so ist möglicherweise die „nationale kommunikative Integration“ in das Migrationsland gar nicht unhinterfragt als der Idealfall anzusehen. Rahmt man die Auseinandersetzung mit Medien und Migration in einem weitergehenden Kontext, so bietet die Vielfalt verschiedener Vernetzungsmomente - von der Herkunftsvernetzung über die bikulturelle bis hin zur transkulturellen Vernetzung - ein breites kommunikatives Integrationspotenzial auf gänzlich unterschiedlichen Ebenen: Möglicherweise sind weltorientierte Migrantinnen und Migranten diejenigen, die sich weit über nationale Grenzen hinaus „,integrieren“ oder zumindest zu einer gewissen kulturellen Verständigung beitragen können.

\section{Literatur}

Adoni, Hanna/Caspi, Dan/Cohen, Akiba (2006): Media, Minorities, and Hybrid Identities: The Arab and Russian Communities in Israel. Cresskill: Hampton Press.

Adoni, Hanna/Cohen, Akiba/Caspi, Dan (2002): The Consumers Choice: Language, Media Consumption and Hybrid Identities of Minorities. In: Communications, 27 (4), S. 411-436. 
Bachmann, Götz/Wittel, Andreas (2006): Medienethnografie. In: Ayaß, Ruth/Bergmann, Jörg (Hrsg.): Qualitative Methoden der Medienforschung. Reinbeck b. Hamburg: Rowohlt, S. 183-219.

Bonfadelli, Heinz/Moser, Heinz (Hrsg.) (2007): Medien und Migration. Europa im multikulturellen Raum? Wiesbaden: VS.

Bucher, Priska/Bonfadelli, Heinz (2007): Mediennutzung von Jugendlichen mit Migrationshintergrund in der Schweiz. In: Bonfadelli, Heinz/Moser, Heinz (Hrsg.): Medien und Migration. Europa im multikulturellen Raum? Wiesbaden: VS, S. 119-145.

Couldry, Nick/Livingstone, Sonia M./Markham, Tim (2007): Media Consumption and Public Engagement. Beyond the Presumption of Attention. Houndmills u. a.: Palgrave.

Dayan, Daniel (1999): Media and Diasporas. In: Gripsrud, Jostein (Hrsg.): Television and Common Knowledge. London, New York: Routledge, S. 18-33.

de Leeuw, Sonja/Rydin, Ingegerd (2007): Diasporic Mediated Spaces. In: Bailey, Olga G./Georgiou, Myria/Harindranath, Ramaswami (Hrsg.): Transnational Lives and the Media: Re-Imagining Diasporas. New York: Palgrave Macmillan, S. 175-194.

Düvel, Caroline (2010): Dimensionen des Medienkulturwandels am Beispiel digitaler Medienaneignung: Kommunikative Vernetzungen russischer Diasporaangehöriger per Mobiltelefon und Internet. In: Hepp, Andreas/Höhn, Marco/Wimmer, Jeffrey (Hrsg.): Medienkulturen im Wandel. Konstanz: UVK (im Druck).

Favell, Adrian (2003): Integration Nations: The Nation-State and Research on Immigrants in Western Europe. In: Comparative Social Research, 22, S. 13-42.

Geertz, Clifford (1997): Dichte Beschreibung. Beiträge zum Verstehen kultureller Systeme. Frankfurt a. M.: Suhrkamp.

Geißler, Rainer/Pöttker, Horst (Hrsg.) (2006): Integration durch Massenmedien/Mass Media-Integration. Bielefeld: Transcript.

Georgiou, Myria (2006): Diaspora, Identity and the Media: Diasporic Transnationalism and Mediated Spatialities. Cresskill: Hampton Press.

Georgiou, Myria (2007): Transnational Crossroads for Media and Diaspora: The Challenges for Research. In: Bailey, Olga G./Georgiou, Myria/Harindranath, Ramaswami (Hrsg.): Transnational Lives and the Media: Re-Imagining Diasporas. New York: Palgrave, S. 11-32.

Glaser, Barney G./Strauss, Anselm L. (1999): Discovery of Grounded Theory: Strategies for Qualitative Research. New Brunswick: AldineTransaction.

Gumpert, Gary/Drucker, Susan J. (2007): Diaspora: An Urban Communication Paradigm. In: Bailey, Olga G./Georgiou, Myria/Harindranth, Ramaswami (Hrsg.): Transnational Lives and the Media: Re-Imagining Diasporas. New York: Palgrave Macmillan, S. 195-211.

Hafez, Kai (2002): Türkische Mediennutzung in Deutschland. Hemmnis oder Chance der gesellschaftlichen Integration? Eine qualitative Studie im Auftrag des Presse- und Informationsamtes der Bundesregierung. Hamburg: DOI.

Hafez, Kai (2004): Die Mediennutzung der Türken und Kurden in Deutschland. Bonn: Navend.

Hall, Stuart (1994): Rassismus und kulturelle Identität. Ausgewählte Schriften 2. Hamburg: Argument Verlag.

Hasebrink, Uwe/Domeyer, Hanna (2010): Zum Wandel von Informationsrepertoires in konvergierenden Medienumgebungen. In: Hartmann, Maren/Hepp, Andreas (Hrsg.): Die Mediatisierung der Alltagswelt. Wiesbaden: VS, S. 49-64.

Hasebrink, Uwe/Popp, Jutta (2006): Media Repertoires as a Result of Selective Media Use. A Conceptual Approach to the Analysis of Patterns of Exposure. In: Communications, 31 (2), S. 369-387.

Hepp, Andreas (2006): Transkulturelle Kommunikation. Konstanz: UVK (UTB).

Hepp, Andreas (2009): Transkulturalität als Perspektive: Überlegungen zu einer vergleichenden empirischen Erforschung von Medienkulturen. In: Forum Qualitative Sozialforschung/Forum: Qualitative Social Research, 10 (1), Art.26, http://nbn-resolving.de/urn:nbn:de:0114fqs0901267 [15.7.2010].

Hepp, Andreas/Bozdag, Cigdem/Suna, Laura (2011): Mediale Migranten: Medienwandel und die kommunikative Vernetzung der Diaspora. Wiesbaden: VS (in Vorbereitung). 
Hess, Sabine/Binder, Jana/Moser, Johannes (Hrsg.) (2009): No Integration?! Kulturwissenschaftliche Beiträge zur Integrationsdebatte in Europa. Münster: Transcript.

Hine, Christine (2000): Virtual Ethnography. London u. a.: Sage.

Hollstein, Betina (2006): Qualitative Methoden und Netzwerkanalyse - ein Widerspruch? In: Hollstein, Betina/Straus, Florian (Hrsg.): Qualitative Netzwerkanalyse. Wiesbaden: VS, S. 11-35.

Klingler, Walter/Kutteroff, Albrecht (2009): Stellenwert und Nutzung der Medien in Migrantenmilieus: Ergebnisse einer repräsentativen Studie. In: Media Perspektiven, 2009 (6), S. 297-307.

Kozinets, Robert (2010): Netnography. Doing Ethnographic Research Online. London u. a.: Sage.

Krotz, Friedrich (2005): Neue Theorien entwickeln. Eine Einführung in die Grounded Theory, die Heuristische Sozialforschung und die Ethnographie anhand von Beispielen aus der Kommunikationsforschung. Köln: Halem.

Krotz, Friedrich (2006): Konnektivität der Medien: Konzepte, Bedingungen und Konsequenzen. In: Hepp, Andreas/Krotz, Friedrich/Moores, Shaun/Winter, Carsten (Hrsg.): Konnektivität, Netzwerk und Fluss. Konzepte gegenwärtiger Medien-, Kommunikations- und Kulturtheorie. Wiesbaden: VS, S. 21-42.

Lotz, A. (2000): Assessing Qualitative Television Audience Research: Incorporating Feminist and Anthropological Theoretical Innovation. In: Communication Theory, 10, S. 447-467.

Marcus, George E. (1995): Ethnography in/of the World System: The Emergence of Multisited Ethnography. In: Annual Review of Anthropology, 24, S. 95-117.

Mau, Steffen (2007): Transnationale Vergesellschaftung. Die Entgrenzung sozialer Lebenswelten. Frankfurt a. M.: Campus.

Meyrowitz, Joshua (1995): Medium Theory. In: Crowley, David J./Mitchell, David (Hrsg.): Communication Theory Today. Cambridge: Polity Press, S. 50-77.

Oswald, Ingrid (2007): Migrationssoziologie. Konstanz: UTB (UVK).

Piga, Andrea (2007): Mediennutzung von Migranten: Ein Forschungsüberblick. In: Bonfadelli, Heinz/Moser, Heinz (Hrsg.): Medien und Migration. Europa im multikulturellen Raum? Wiesbaden: VS, S. 209-234.

Pöttker, Horst (2005): Soziale Integration. Ein Schlüsselbegriff für die Forschung über Medien und ethnische Minderheiten. In: Geißler, Rainer/Pöttker, Horst (Hrsg.): Massenmedien und die Integration ethnischer Minderheiten in Deutschland. Bielefeld: Transcript, S. 25-43.

Pries, Ludger (1998): Transnationale soziale Räume. Theoretisch-empirische Skizze am Beispiel der Arbeitswanderbewegungen Mexiko - USA. In: Beck, Ulrich (Hrsg.): Perspektiven der Weltgesellschaft. Frankfurt a. M.: Suhrkamp, S. 55-86.

Pries, Ludger (2008): Die Transnationalisierung der sozialen Welt. Sozialräume jenseits von Nationalgesellschaften. Frankfurt a. M.: Suhrkamp.

Robins, Kevin (2006): The Challenge of Transcultural Diversities: Final Report of the Transversal Study on the Theme of Cultural Policy and Cultural Diversity. In: Robins, Kevin (Hrsg.): The Challenge of Transcultural Diversities. Strassbourg: Council of Europe, S. 7-48.

Robins, Kevin/Aksoy, Asu (2006): Thinking Experience: Transnational Media and Migrant's minds. In: Curran, James/Morley, David (Hrsg.): Media and Cultural Theory. London u. a.: Routledge, S. 86-99.

Schatz, Heribert/Holtz-Bacha, Christina/Nieland, Jörg-Uwe (Hrsg.) (2000): Migranten und Medien. Neue Herausforderungen an die Integrationsfunktion von Presse und Rundfunk. Opladen: Westdeutscher Verlag.

Simon, Erk (2007): Migranten und Medien 2007. Zielsetzung, Konzeption und Basisdaten einer repräsentativen Studie der ARD/ZDF-Medienkommission. In: Media Perspektiven, 2007 (9), S. 426-435.

Trebbe, Joachim (2007): Types of Integration, Acculturation Strategies and Media Use of Young Turks in Germany. In: Communications: the European Journal of Communication Research, 32, S. 171-191.

Weber, Max (1972): Wirtschaft und Gesellschaft. Grundriss der verstehenden Soziologie. Tübingen: Mohr Verlag. 
Weiß, Hans-Jürgen/Trebbe, Joachim (2001): Mediennutzung und Integration der türkischen Bevölkerung in Deutschland. Ergebnisse einer Umfrage des Presse- und Informationsdienstes der Bundesregierung. Potsdam: GöfaK Medienforschung GmbH.

\section{Unverzichtbar!}

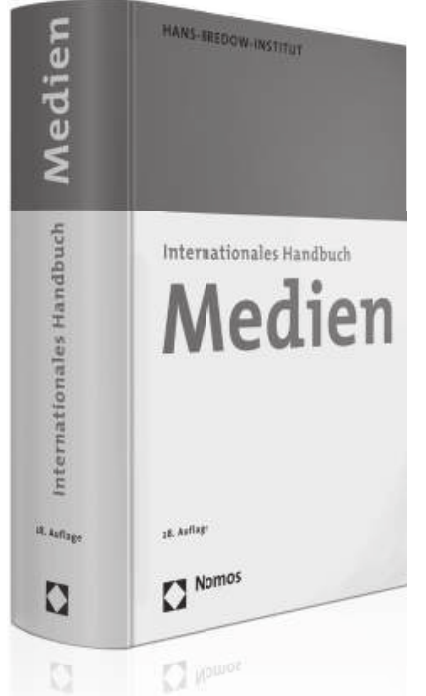

Internationales Handbuch Medien

Vom Hans-Bredow-Institut

28. Auflage 2009,

1.308 S., geb., 128,- $€$,

ISBN 978-3-8329-3423-1
"Medienschaffenden, die international interessiert sind oder sich vor einem Auslandsaufenthalt mit dem Mediensystem ihres Gastlandes vertraut machen möchten, ist dieses Handbuch daher wärmstens ans Herz zu legen." Jan Fees, MedienWirtschaft 2/10

"seit vielen Jahren ein unentbehrliches Nachschlagewerk in allen einschlägigen Fachbibliotheken und hoffentlich auch in allen größeren Allgemeinbibliotheken. Seine nationalen und internationalen Informationen sind als Ausgangspunkt für alle eingehenderen Recherchen über nationale Mediensysteme und dortige Medien unverzichtbar."

Wilbert Ubbens, www.ifb.bsz-bw.de März 2010

Bitte bestellen Sie im Buchhandel oder versandkostenfrei unter $\downarrow$ www.nomos-shop.de

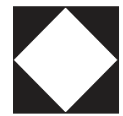

Nomos 